\title{
Análise normativa dos comitês de investigação de óbitos, de controle de infecção e sua interface com a segurança do paciente em organizações complexas
}

Normative analysis of the death investigation committees, infection control and their interface with patient safety in complex organizations

Análisis normativo de los comités de investigación de las muertes, del control de la infección y su interacción con la seguridad del paciente en las organizaciones complejas

\section{Eduardo André Viana Alves ${ }^{1}$}

Resumo: A segurança do paciente entrou como tema obrigatório para os serviços de saúde brasileiros com a formalização do Núcleo de Segurança do Paciente. Contudo, as organizações hospitalares já dispõem de comitês e comissões que também trabalham o risco sanitário e os eventos adversos em objetos específicos como as comissões de controle de infecção hospitalar e os comitês de investigação de óbito. O objetivo deste trabalho é analisar os regulamentos sanitários que criaram os comitês de investigação de óbitos e as comissões de controle de infecção hospitalares comparando e observando em que ponto eles interagem com o Núcleo de Segurança do Paciente em organizações complexas. Realizou-se análise documental dos ordenamentos normativos brasileiros que criaram estes comitês. Os resultados foram sistematizados de acordo com os seguintes parâmetros: nome da instância, normatização de criação, grau de participação, composição da equipe, forma de trabalho, instrumentos de trabalho, mecanismos de fiscalização regulação e controle. Observa-se que estas instâncias atuam dentro dos serviços de saúde interagindo em grau variado com a segurança do paciente. Essas instâncias apesar de receberem diversas denominações como 'Comitês', 'Comissões' ou 'Núcleos', mantém como ponto comum a obrigatoriedade de sua existência pelos regulamentos sanitários. Verifica-se, ainda, o risco de perda de eficiência devido à sobreposição de objetos de trabalho em um contexto de complexidade do sistema organizacional hospitalar. Contudo, nota-se uma intensa capacidade de atuação sinérgica, se estes comitês trabalharem a fim de atingir objetivos comuns o que levaria a criação de uma inteligência coorporativa institucional.

Palavras-chave: Segurança do Paciente, Serviços de Saúde, Legislação Sanitária

\begin{abstract}
Patient safety entered as a mandatory subject for the Brazilian public health services with the formalization of the Patient Safety Unit. However, hospital organizations already have committees and commissions that also work on the health risk and adverse events on specific objects such as of hospital infection control committees and the death investigation committees. The objective of this study is to analyze sanitary regulations that created the death investigation committees and hospital infection control committees comparing and observing at which point they interact with the Patient Safety Unit in complex organizations. Documentary analysis was carried out of the Brazilian regulatory frameworks which created these committees. The results were organized according to the following parameters: instance name, creation of standardization, degree of participation,

\footnotetext{
1 Médico Sanitarista da Secretaria de Saúde do Distrito Federal, Especialista em Regulação e Vigilância Sanitária da Agência Nacional de Vigilância Sanitária - Anvisa. Brasília - Distrito Federal. Brasil. E-mail: eduardo.dr@gmail.com
} 
team composition, way of working, work tools, monitoring, regulation and control mechanisms. It is observed that these bodies act within the health services interacting in varied degree with patient safety. These instances despite receiving several denominations as 'committees', or 'cores', keep in common requirement of their existence by sanitary regulations. There is also the risk of loss of efficiency due to overlapping of work objects in a context of organizational complexity of the hospital system. However, there is an intense ability to synergistic action if these committees work in order to achieve common goals that would lead to the creation of an institutional corporative intelligence.

Keywords: Patient safety, Health services, Health legislation.

Resumen: La seguridad del paciente fue una asignatura obligatoria para los servicios de salud brasileños con la formalización del Centro de Seguridad del Paciente. Sin embargo, las organizaciones hospitalarias ya tienen comités y comisiones que también trabajan el riesgo para la salud y los eventos adversos en los objetos específicos, como las comisiones de control de infección hospitalaria y las comisiones de investigación de la muerte. El objetivo de este estudio es analizar las normas sanitarias que crearon las comisiones de investigación de la muerte y de los comités de control de infección hospitalaria comparando y observando en ese momento en que interactúan con la Unidad de Seguridad del Paciente en organizaciones complejas. Análisis documental Held de los sistemas reguladores brasileños que han creado estos comités. Los resultados fueron organizados de acuerdo a los siguientes parámetros: nombre de la instancia, la creación de la normalización, el grado de participación, la composición del equipo, forma de trabajo, herramientas, mecanismos de control de supervisión y regulación. Se observa que estos cuerpos actúan dentro de los servicios de salud interactúan en diversos grados con la seguridad del paciente. Estos casos a pesar de recibir varias denominaciones como "comités", "comisiones" o "núcleos", mantiene en común que la exigencia de su existencia por las regulaciones sanitarias. También existe el riesgo de pérdida de eficiencia debido a la superposición de los objetos de trabajo en un contexto de complejidad organizativa del sistema hospitalario. Sin embargo, hay una intensa capacidad de acción sinérgica si estos comités trabajan con el fin de alcanzar objetivos comunes que conduzcan a la creación de una inteligencia corporativa institucional

Palabras-Ilave: Seguridad del paciente, Servicios de salud, Legislación sanitaria

\section{Introdução}

Os hospitais são organizações complexas. Segundo Osmo (1):

A organização hospitalar é uma das mais complexas devido a coexistência de inúmeros processos assistenciais e administrativos, diversas linhas de produção simultâneas e uma fragmentação de processos de decisão assistencial com presença de equipe multiprofissional e multiprofissional com elevado grau de autonomia. Para tanto utiliza tecnologia de forma intensiva e extensiva. Podendo ainda constituir-se em espaço de ensino e aprendizagem além de campo de produção científica. 
Cada um desses fatores incrementa ao processo assistencial um risco que somado tem a potencialidade de expor ao paciente uma série de eventos adversos que podem evoluir do dano leve ao óbito.

Assim, a realidade dos serviços mais complexos mostra a existência de uma gama de instâncias que estão envolvidas no monitoramento e regulação de uma série de produtos, desfechos e processos considerados críticos ao procedimento assistencial e que executam funções que guardam relação com a segurança do paciente.

Este trabalho parte da premissa que o arcabouço normativo sanitário brasileiro que trata do funcionamento dos serviços de saúde procurou adequar esta realidade complexa, de forma a mitigar e eliminar os riscos existentes nos estabelecimentos assistenciais de saúde.

A partir desta premissa surge o questionamento: Qual a relação da segurança do paciente com os demais comitês e comissões? Como efetivar a segurança do paciente dentro da realidade de organizações complexas de saúde como os hospitais?

Este artigo analisará o Núcleo de Segurança do Paciente (NSP), os Comitês de Investigação de Óbitos e a Comissão de Controle de Infecção Hospitalar (CCIH). O tema ganha importância dentro do Direito Sanitário ao responsabilizar os gestores e profissionais de saúde por serviços mais seguros e de maior qualidade ao paciente. Espera-se, dessa forma, auxiliar demais instituições na elaboração de estratégias em prol da segurança do paciente através do conhecimento da existência e importância desses comitês relacionados direta ou indiretamente com o tema.

\section{Objetivo}

O objetivo deste trabalho é analisar os regulamentos sanitários que criaram os comitês de investigação de óbitos e comitês de controle de infecção hospitalares comparando e observando em que ponto eles interagem com o Núcleo de Segurança do Paciente em organizações complexas.

\section{Metodologia}

Realizou-se análise documental dos ordenamentos normativos brasileiros que criaram estes comitês. Foram analisadas normatizações de órgãos reguladores nacionais 
como Ministério da Saúde, Agência Nacional de Vigilância Sanitária (ANVISA) e Congresso Nacional.

Os resultados foram sistematizados em tabela de acordo com os seguintes parâmetros: nome da instância, normatização de criação, grau de participação, composição da equipe, forma de trabalho, instrumentos de trabalho, mecanismos de fiscalização regulação e controle.

\section{Resultados}

A normatização que cria o Núcleo de Segurança do Paciente é aplicada a quase todos os serviços de saúde, desde os mais simples aos mais complexos. O NSP foi instituído através de resolução específica da Anvisa (RDC Anvisa n 36/2013) que criou os Núcleos de Segurança do Paciente, definiu sua composição e objetivos, obrigou a formulação de um plano de segurança do paciente pelos serviços de saúde, e estabeleceu os mecanismos de notificação e investigação de eventos adversos.

$O$ objeto de trabalho principal da segurança do paciente é o evento adverso. Assim o NSP busca a prevenção, identificação, mitigação e análise do evento adverso. A RDC Anvisa $n^{\circ} 36 / 2013$ conceitua evento adverso, incidente e danos. Evento adverso é o incidente que resulta em danos à saúde. Incidente é o evento ou circunstância que poderia ter resultado, ou resultou, em dano desnecessário à saúde. Dano é o comprometimento da estrutura ou função do corpo e/ou qualquer efeito dele oriundo, incluindo doenças, lesão, sofrimento, morte, incapacidade ou disfunção, podendo, assim, ser físico, social ou psicológico (2).

Observa-se que estes conceitos perpassam outros objetos de trabalho dos demais comitês em especial o óbito e a infecção relacionada a serviços de saúde².

A Comissão de Controle de Infecção Hospitalar apresenta um dos mais robustos arcabouços normativos, sendo prevista inclusive em lei específica (Lei ํo 9.431 de 6 de janeiro de 1997) (3). No ano seguinte a CCIH foi regulamentada por portaria específica no Ministério da Saúde (4).

2 O termo "infecção hospitalar" é frequentemente substituído por "infecções relacionadas aos serviços de saúde" de forma a abranger as infecções relacionadas ao processo assistencial em outros serviços de saúde. Contudo para este artigo serão considerados como sinônimos. 
Trata-se de uma comissão que é responsável pelo monitoramento e pela adoção de estratégias de um desfecho/evento adverso desfavorável ocorrido nos serviços de saúde sendo um indicador de processo de trabalho da qualidade assistencial.

A $\mathrm{CCIH}$ apresenta caráter deliberativo dentro da instituição. Encontra-se classicamente envolvida: com os eventos adversos infecciosos e sua prevenção, com o uso racional de antimicrobianos, com o monitoramento da resistência microbiana e com as campanhas de higienização das mãos.

Segundo Wachter (5):

Se considerarmos as IHs como resultado da falha de adesão às práticas baseadas em evidências para a prevenção de eventos adversos (postura na qual acredito), então as IHs poderão ser a fonte mais comum de danos sérios e evitáveis nos cuidados em saúde.

Neste aspecto a CCIH apresenta forte vínculo com o NSP tanto em relação ao objeto de trabalho (evento adverso de causa infecciosa) quanto em relação aos métodos e trabalho como a investigação de eventos adversos, redução de danos utilização de campanhas de prevenção (como as campanhas de higienização das mãos) (5).

O monitoramento das infecções permite mensurar um indicador de qualidade do serviço de saúde. A infecção nestes casos representa um evento não esperado no processo assistencial, principalmente quando relacionado as cirurgias limpas. Neste caso serve como um indicador para quebra de protocolos como os da higienização de mãos, superfícies ou campos cirúrgicos. A CCIH monitora também a resistência microbiana, que tem contribuído com eventos adversos graves como o óbito de pacientes por impossibilidade de opções terapêuticas para o tratamento de infecções. Neste caso, a $\mathrm{CCIH}$ é responsável por adotar estratégias de uso racional de antimicrobianos nos servidos de saúde de forma a minimizar o risco de desenvolvimento de resistências bacterianas.

Os comitês hospitalares de análise de óbitos maternos, fetais e neonatais tornaramse obrigatórios para todos os serviços de atenção materno e neonatal através da RDC Anvisa $n^{\circ} 36 / 2008$ (6). Nos demais serviços estes comitês não são obrigatórios, mas os serviços necessitam realizar atividades de vigilância de óbitos e prestar auxílio às equipes de investigação dos comitês municipais de óbitos. Em 2008, definiu-se a obrigatoriedade de busca ativa de óbitos maternos pelo Núcleo Hospitalar de Epidemiologia que são responsáveis por esta atividade nos serviços onde os comitês não estão presentes (7). Em 
2010, a vigilância do óbito infantil e fetal tornou-se obrigatória nos serviços de saúde (públicos e privados) que integram o Sistema Único de Saúde (8).

Os comitês de investigação de óbitos apresentam um sistema de investigação com diversos questionários a serem preenchidos por diversos entes do sistema de maneira a construir um retrato completo da causa o óbito. Os comitês possuem forte vínculo com o sistema de informação de mortalidade podendo inclusive alterar a causa básica do óbito ao final do processo de investigação (9) (10).

O monitoramento dos óbitos permite mensurar um indicador de qualidade do serviço de saúde embora a comparação de taxas de mortalidade entre serviços seja uma tarefa complexa, devido à existência de múltiplos parâmetros relacionados à doença, ao paciente, ao serviço e ao sistema de saúde ao qual o serviço está vinculado que influenciam no resultado final do atendimento, como: o perfil populacional, gravidade da doença no momento do diagnóstico, o nível assistencial do serviço de saúde, entre outros.

O estudo dos óbitos permite comparar serviços ou regiões se estiverem devidamente estratificados por grupos de pacientes. Assim a depender da característica do serviço como as características epidemiológicas da população atendida e sua demanda os comitês podem ser subdivididos em subcomitês específicos como o Comitê de Investigação de Óbitos Fetal e Neonatal.

O Comitê de Investigação de Óbitos Fetal e Neonatal verifica as causas que contribuíram para a ocorrência do óbito, classifica essas causas em evitáveis ou não evitáveis e sugere melhorias nos processos de trabalho para a prevenção de novos óbitos em situações semelhantes. Entre as diversas causas de óbito investigadas estão aquelas relacionadas a eventos adversos. Esta faixa etária em especial é mais dependente das tecnologias de saúde, tendo esta característica reflexo direto na segurança do paciente.

O Ministério da Saúde, no Manual de Vigilância do Óbito Infantil e Fetal e do Comitê de Prevenção do Óbito Infantil e Fetal (10), destaca a importância do estudo da mortalidade perinatal:

A mortalidade perinatal - óbitos fetais e neonatais precoces com peso ao nascer a partir de $500 \mathrm{~g}$ e/ou 22 semanas de idade gestacional - tem sido recomendada como 0 indicador mais apropriado para a análise da assistência obstétrica e neonatal e de utilização dos serviços de saúde, de modo a dar visibilidade ao problema e propiciar a identificação das ações de prevenção para o alcance de ganhos mútuos na redução das mortes fetal e neonatal precoce evitáveis. 
Dessa forma na investigação dos óbitos fetais são excluídos os abortos, em produtos de gestação menores de 500 gramas ou menores de $25 \mathrm{~cm}$ ou com idade gestacional menor que 22 semanas.

Os óbitos que preencham esses critérios são investigados e, ao final da investigação, são classificados frente aos critérios de evitabilidade.

O Comitê de Investigação de Óbitos Acima de 28 dias verifica as causas de óbitos em bebês e crianças. Os óbitos pós-neonatais são menos sensíveis a atenção direta dos serviços de saúde em relação aos óbitos neonatais. A medida que as crianças ficam mais velhas, a causa básica do óbito acaba sendo mais influenciada por fatores relacionados a baixos níveis de saúde e de desenvolvimento socioeconômico (11).

O Comitê de Óbitos Maternos investiga todos os óbitos de mulheres em idade fértil observando sua relação com a gestação. Os problemas relacionados à assistência materna impactam sobre esse grupo têm reflexo nas ações de segurança do paciente. Os óbitos maternos são bem menos comuns que os óbitos infantis, mesmo assim o Brasil não tem conseguido alcançar sua meta de redução da mortalidade materna, assim discute-se como estratégia a ampliação da atuação deste comitê para o estudo dos óbitos e dos quase óbitos ocorridos no serviço para este estrato da população a fim de entender os fatores desencadeadores e contribuintes para o óbito de forma que o Comitê possa indicar novas estratégias para que esses eventos não ocorram (12).

O óbito para o NSP é um evento adverso grave. A RDC Anvisa n 36/2013 estabelece um fluxo específico para a notificação e investigação do óbito, pois o entende como um indicador de saúde extremamente significativo. Esta característica o aproxima dos comitês hospitalares de óbito. Contudo, nem todo óbito é decorrente de um evento adverso. Existem causas naturais de óbito que não são foco do NSP, mas que são foco desses comitês. Dessa forma, torna-se necessário estabelecer os pontos comuns e as diferenças entre esses objetos de trabalho.

Após a apresentação geral, as informações dos três comitês foram consolidadas e apresentadas a seguir:

\section{- Sobre o objeto/escopo de trabalho}

Um ponto comum a todos os comitês é sua interface com o tema da segurança do paciente, abordando-o, às vezes como um evento adverso, às vezes como parte do 
processo de trabalho (prevenção de infecções), ou restringindo seu campo de observação a um evento específico em um grupo populacional (óbitos em mulheres em idade fértil, óbitos em crianças de acordo com a faixa etária específica).

\section{- Sobre a normatização de criação}

A CCIH é o único comitê entre os três estudados que apresenta previsão em lei federal específica (3). Os comitês de investigação de óbito são induzidos por Portaria do Ministério da Saúde que vinculam a existência desses comitês a repasses financeiros (13) (14) e a resolução da Anvisa que relaciona a não existência, ou o não oferecimento por parte do gestor das condições de funcionamento desses comitês, a configuração de infração sanitária aos quais os gestores estão sujeitos (15).

\section{- Sobre o grau de participação}

O grau de participação do comitê nas esferas decisórias do serviço de saúde influencia na possibilidade de uma maior intervenção sobre os problemas encontrados. Observa-se neste caso que o NSP e a CCIH apresentam maior poder para a realização das intervenções que julgarem necessárias, pois participam das instâncias colegiadas de suas instituições. A atuação dos comitês de mortalidade é consultiva dentro dos serviços de saúde.

\section{- Sobre a composição da equipe}

Estes comitês apresentam composição multiprofissional. Este desenho permite a uma melhor compreensão dos problemas encontrados assim como possibilitaria uma intervenção mais eficaz sobre os mesmos. Contudo, vale recordar que a quantidade de profissionais existentes nos serviços de saúde é finita, o que pode levar a sobreposição de atividades de um mesmo profissional em diversos comitês. A portaria que regulamenta o funcionamento da $\mathrm{CCIH}$ além de definir os integrantes que devem compor a comissão regulamenta também objetivamente a carga horária mínima necessária a esses profissionais que utiliza como parâmetro a quantidade de leitos existentes no estabelecimento (4). Para os comitês de óbito há apenas recomendação em manual do ministério da saúde (9) (10). Para o NSP a RDC Anvisa $n^{\circ} 36 / 2013$ define que a equipe deve ser compatível com as atividades que 
desempenha, desta forma a RDC insere um fator subjetivo a norma que deverá ser observado pelos órgãos reguladores no momento de fiscalização (2).

\section{- Sobre a forma de trabalho}

Todos os comitês utilizam o raciocínio epidemiológico, juntamente com seus instrumentos agregados. Também, podem realizar treinamento e capacitação de equipes, utilizar padronizações com normatização de condutas, divulgar informes dos resultados do trabalho.

\section{- Sobre os instrumentos de trabalho}

Observa-se que a $\mathrm{CCIH}$ e os comitês de óbito apresentam instrumentos testados, como: Fichas de notificação ou declarações (Declarações de óbito), Fichas de investigação (de óbitos, de surtos ou eventos adversos), Investigação laboratorial de resistência microbiana (mais presente na $\mathrm{CCIH}$ ). O NSP ainda se encontra validando instrumentos específicos como fichas de investigação e notificação. Para notificação e investigação utiliza sistema web da Anvisa.

\section{- Sobre a fiscalização regulação e controle}

Devido a sua importância estratégica a identificação e atuação preventiva e corretiva sobre os riscos envolvidos em suas linhas de atuação, os três comitês apresentam instâncias extra-hospitalares dentro das secretarias de saúde municipais, estaduais e do governo federal responsáveis pela supervisão ou fiscalização dos comitês hospitalares de cada sistema local de saúde. Os pontos apresentados foram consolidados no quadro 1 : 
Quadro 1 - Quadro comparativo entre os Comitês de Hospitalares de Investigação de Óbitos, Comitê de Controle de Infecção Hospitalar - CCIH e o Núcleo de Segurança do Paciente - NSP.

\begin{tabular}{|c|c|c|c|c|c|c|}
\hline $\begin{array}{l}\text { Nome do } \\
\text { Comitê }\end{array}$ & $\begin{array}{l}\text { Normatização } \\
\text { de criação }\end{array}$ & $\begin{array}{c}\text { Grau de } \\
\text { participação }\end{array}$ & $\begin{array}{l}\text { Composição da } \\
\text { equipe }\end{array}$ & $\begin{array}{l}\text { Forma de } \\
\text { trabalho }\end{array}$ & $\begin{array}{l}\text { Instrumentos de } \\
\text { trabalho }\end{array}$ & $\begin{array}{l}\text { Fiscalização } \\
\text { Regulação e } \\
\text { Controle }\end{array}$ \\
\hline $\begin{array}{l}\text { Comitês de } \\
\text { Hospitalares } \\
\text { de } \\
\text { Investigação } \\
\text { de Óbitos }\end{array}$ & $\begin{array}{l}\text { Portaria do } \\
\text { Ministério da } \\
\text { Saúde } \\
\text { Resolução da } \\
\text { ANVISA }\end{array}$ & Consultivo & \multirow{3}{*}{ Multiprofissional } & \multirow{3}{*}{$\begin{array}{c}\text { Vigilância } \\
\text { epidemiológica } \\
\text { Treinamento e } \\
\text { capacitação de } \\
\text { equipes } \\
\text { Uso de } \\
\text { normatização de } \\
\text { condutas } \\
\text { Uso de informes } \\
\text { para divulgação } \\
\text { dos resultados } \\
\text { do trabalho }\end{array}$} & \multirow{3}{*}{$\begin{array}{c}\text { Fichas de } \\
\text { notificação ou } \\
\text { declarações } \\
\text { (Declarações de } \\
\text { óbito), } \\
\text { Fichas de } \\
\text { investigação (de } \\
\text { óbitos, de surtos } \\
\text { ou eventos } \\
\text { adversos) } \\
\text { Investigação } \\
\text { laboratorial de } \\
\text { resistência } \\
\text { microbiana } \\
\text { (mais presente } \\
\text { na CCIH) }\end{array}$} & \multirow{3}{*}{$\begin{array}{c}\text { Instâncias } \\
\text { extra- } \\
\text { hospitalares } \\
\text { responsáveis } \\
\text { pela } \\
\text { supervisão ou } \\
\text { fiscalização } \\
\text { dos comitês } \\
\text { hospitalares } \\
\text { de cada região } \\
\text { administrativa }\end{array}$} \\
\hline $\begin{array}{l}\text { Comitê de } \\
\text { Controle de } \\
\text { Infecção } \\
\text { Hospitalar - } \\
\text { CCIH }\end{array}$ & $\begin{array}{l}\text { Lei Federal } \\
\text { Portaria do } \\
\text { Ministério da } \\
\text { Saúde }\end{array}$ & Deliberativo & & & & \\
\hline $\begin{array}{l}\text { Núcleo de } \\
\text { Segurança do } \\
\text { Paciente - } \\
\text { NSP }\end{array}$ & $\begin{array}{l}\text { Resolução da } \\
\text { ANVISA }\end{array}$ & Deliberativo & & & & \\
\hline
\end{tabular}

\section{Discussões}

A divisão das instâncias administrativas de gerenciamento dos riscos nos hospitais requer uma discussão prévia sobre os sistemas complexos e o papel dos comitês como forma de intervenção sobre esses sistemas.

Wachter, R.M. (5) apresenta a teoria da complexidade como:

(...) um ramo do pensamento de gestão que coloca que grandes organizações não funcionam como máquinas previsíveis e estáticas, em que as Entradas A e B previsivelmente levam para o Resultado $\mathrm{C}$. Em vez disso, elas operam como sistemas adaptativos complexos, com elementos de imprevisibilidade, codependência e sem linearidade, que devem ser analisados enquanto tentamos fazê-los funcionar melhor.

Fragata et al (16) ao abordarem os cuidados de saúde e os sistemas complexos, os comparam com os sistemas mecânicos onde o sistema automotor pode ser decomposto em partes, estas partes melhoradas e depois remontadas. Sobre os sistemas complexos fazem a seguinte ponderação:

Esse processo de decomposição hierárquica funciona bem para sistemas mecânicos, mas não para sistemas de complexidade dinâmica, porque, por serem não lineares, não têm pontos de equilíbrio, parecendo antes aleatórios e caóticos; esses sistemas são constituídos por muitos agentes (médicos, enfermeiros, pacientes, pagadores, gestores...) que tendem a atuar dentro de redes profissionais e sociais, mas também independentemente, por interesses próprios e diferentes, não raras vezes conflitantes. 
Assim qual o melhor modelo de abordagem da segurança do paciente em serviços de saúde complexos? Utilizar um modelo tradicional com forte base hierárquica e, portanto, a criação de um nicho específico dentro do organograma institucional para a segurança do paciente ou utilizar uma abordagem dinâmica, onde a hierarquia apresenta ordem consensual?

O modelo brasileiro optou pela formalização do NSP através da obrigatoriedade de sua existência no ordenamento sanitário federal. Wachter (5) ao analisar o papel da regulação pondera:

Uma lei ou um regulamento podem facilmente parecer a maneira correta de solucionar o importante problema da segurança do paciente, mas nós nunca devemos cometer o erro de subestimar a confusão e a complexidade de nosso mundo.

Contudo Vincent (17) ao discutir a liderança por meio da política e da regulamentação contra-argumenta:

Muitos dos mais potentes estímulos para a segurança do paciente estão fora das instituições de atendimento médico-hospitalar. Relatórios do governo e de organizações profissionais, bem como a pressão social, legal e da mídia, contribuem para o surgimento da segurança do paciente como tema atual.

Este autor pondera também que os resultados são alcançados de forma lenta, mas são vitais para a manutenção do interesse da segurança do paciente (17). Assim destaca a importância e necessidade de um agente regulador externo induzir a discussão e introjeção da segurança do paciente dentro dos serviços de saúde.

A cultura organizacional brasileira talvez explique a necessidade da existência da formalização de leis, instâncias e mecanismos de fiscalização e de obrigação para que a segurança do paciente seja implementada pelos serviços de saúde.

Dessa forma é inegável o poder dado ao NSP ao responsabilizá-lo pelas ações de segurança do paciente dentro da instituição. Compreendendo a complexidade do sistema hospitalar pergunta-se: Como organizar o NSP em relação aos demais comitês existentes? A RDC Anvisa $n^{\circ} 36 / 2013$ nos dá orientações sobre o funcionamento do NSP em relação aos comitês (art. $4^{\circ}, \S 1^{\circ}$ ):

A direção do serviço de saúde pode utilizar a estrutura de comitês, comissões, gerências, coordenações ou núcleos já existentes para o desempenho das atribuições do NSP. 
Dessa forma a RDC permite ao gestor decidir se as atividades do NSP serão executadas por outros comitês ou se será necessário a criação de uma estrutura própria para este fim.

A aplicação prática da interface dos comitês em um evento adverso evidencia realmente a complexidade do sistema de saúde. Tomemos como exemplo a investigação do óbito de uma gestante pós-cesárea devido a uma infecção hospitalar. As ações realizadas no serviço de saúde envolveriam necessariamente o NSP, a CCIH e o comitê de investigação de óbito. Em um sistema mecânico a proposta de trabalho das equipes seria simples.

Cada comitê faria sua parte e a soma das partes (investigação, relatórios e intervenção) levaria a instituição a adotar mecanismos mais seguros de prevenção de eventos adversos para estes casos específicos, com eficácia e eficiência organizacional. Contudo, como já apresentado, o sistema hospitalar é complexo, e, portanto, os desfechos possíveis são variados. Além do desfecho acima descrito diversos cenários podem ser possíveis, dentre eles:

- Todos os comitês fazerem tudo. Os comitês trabalham separadamente utilizando todos os instrumentos disponíveis, inclusive dos outros comitês. O resultado final seriam relatórios fragmentados e sobrepostos, e embora o resultado final possa levar a um sistema mais eficaz e seguro, os recursos utilizados tornaram o resultado ineficiente.

- Um comitê faz tudo. Neste cenário apenas um comitê se responsabiliza por investigar, propor e executar as ações relacionadas ao desdobramento do óbito. Além da sobrecarga de trabalho dada a apenas um comitê, mostra-se falhas na gestão das demais equipes que se tornaram ociosas.

- Ninguém faz nada. Neste cenário cada comitê aguarda que o outro execute suas atividades e não toma a iniciativa de fazê-lo. Como resultado final, temos um sistema ineficaz, ineficiente e passível de repetição do mesmo evento na instituição, porque as medidas preventivas não foram adotadas.

Conforme ponderado, observa-se que as ações dos comitês não devem ser meramente burocráticas e cartoriais, pois assim não abrangerão a complexidade da dinâmica do cuidado da saúde. 
As ações de segurança do paciente não poderão jamais serem colocadas a cargo exclusivo do NSP sob pena de sobrecarregá-lo e restringir suas ações ao preenchimento de condições de funcionamento obrigatórias por normatização. Estas ações devem ser realizadas em todas as instâncias da instituição. Sendo assim os demais comitês terão papel fundamental na promoção e ampliação destas ações de acordo com o seu grau de especificidade.

Observa-se que a $\mathrm{CCIH}$ apresenta forte vínculo com o NSP tanto em relação ao objeto de trabalho (evento adverso de causa infecciosa) quanto em relação aos métodos e trabalho como a investigação de eventos adversos, redução de danos utilização de campanhas de prevenção (como as campanhas de higienização das mãos).

Observa-se que os comitês de investigação de óbito apresentam método e instrumental robustos que permitem através da aplicação de protocolos específicos identificar a evitabilidade ou não do óbito.

O Núcleo de Segurança do Paciente é a mais nova comissão incorporada aos serviços de saúde entre os três estudados. Por essa característica pôde absorver não só todo o arcabouço teórico específico sobre o tema, mas também pôde operacionalmente absorver a essência das normativas, das metodologias e dos instrumentos de trabalho desses outros comitês. As ações de segurança do paciente também não se iniciam com a criação do NSP, assim como os instrumentos de investigação e controle dos eventos adversos não foram criados sem observar os demais instrumentos existentes.

\section{Conclusões}

Observa-se que estas instâncias atuam dentro dos serviços de saúde interagindo em grau variado com a segurança do paciente. Essas instâncias apesar de receberem diversas denominações como 'Comitês', 'Comissões' ou 'Núcleos', mantém como ponto comum a obrigatoriedade de sua existência pelos regulamentos sanitários.

Os Comitês de Hospitalares de Investigação de Óbitos (materno, fetal e infantil), a Comissão de Controle de Infecção Hospitalar e o Núcleo de Segurança do Paciente analisam dois fortes desfechos clínicos que impactam na qualidade da atenção prestada: o óbito e a ocorrência de infecção durante a internação.

Observa-se o risco de perda de eficiência devido a sobreposição de objetos de trabalho em um contexto de complexidade do sistema organizacional hospitalar. Contudo 
observa-se uma intensa capacidade de atuação sinérgica, se estes comitês trabalharem a fim de atingir objetivos comuns o que levaria a criação de uma inteligência coorporativa institucional.

\section{Referências}

1 - Osmo AA. Processo gerencial. In: Vecina Neto G, Malik AM. Gestão em saúde. Rio de Janeiro: Guanabara Koogan; 2011.

2 - Brasil. Agência Nacional de Vigilância Sanitária. Resolução da Diretoria Colegiada RDC no 36, de 25 de julho de 2013. Institui ações para a segurança do paciente em serviços de saúde e dá outras providências. Brasília. [Acesso em 19 set 2015]. Disponível em: http://bvsms.saude.gov.br/bvs/saudelegis/anvisa/2013/rdc0036 2507 2013.pdf

3 - Brasil. Lei no 9.431 de 6 de janeiro de 1997. Dispõe sobre a obrigatoriedade da manutenção de programa de controle de infecções hospitalares pelos hospitais do País. Brasília. [Acesso em 19 set 2015]. Disponível em: http://www.planalto.gov.br/ccivil 03/Leis/L9431.htm

4 - Brasil. Ministério da Saúde. Portaria n².616, de 12 de maio de 1998. Dispõe sobre as diretrizes e normas para a prevenção e o controle das infecções hospitalares. Brasília. [Acesso em 19 set 2015]. Disponível em: http://portal.anvisa.gov.br/wps/wcm/connect/8c6cac8047457a6886d6d63fbc4c6735/PORT ARIA+N\%C2\%B0+2.616,+DE+12+DE+MAIO+DE+1998.pdf?MOD=AJPERES

5 - Wachter RM. Compreendendo a segurança do paciente. Porto Alegre: AMGH; 2013.

6 - Brasil. Agência Nacional De Vigilância Sanitária. Resolução da Diretoria Colegiada $\mathrm{RDC} \mathrm{n}^{\circ}$ 36, de 3 de junho de 2008. Dispõe sobre Regulamento Técnico para Funcionamento dos Serviços de Atenção Obstétrica e Neonatal. Diário Oficial da União Brasília, 04 jun. 2008. [Acesso em 19 set 2015]. Disponível em:

http://www20.anvisa.gov.br/segurancadopaciente/index.php/legislacao/item/rdc-n-36-de-03de-junho-de-2008

7 - Brasil. Ministério Da Saúde. Portaria GM n 1.119 de 05 de junho de 2008. Regulamenta a Vigilância de Óbitos Maternos. Brasília. [Acesso em 03 dez 2015]. Disponível em http://www.saude.pr.gov.br/arquivos/File/Portaria1119obitosmaternos2008.pdf.

8 - Brasil. Ministério da Saúde. Portaria no 72/GM/MS, de 11 de janeiro de 2010. Estabelece que a vigilância do óbito infantil e fetal é obrigatória nos serviços de saúde (públicos e privados) que integram o Sistema Único de Saúde (SUS). Brasília. [Acesso em 19 set 2015]. Disponível em:

http://bvsms.saude.gov.br/bvs/saudelegis/gm/2010/prt0072 1101 2010.html 
9 - Brasil. Ministério da Saúde. Secretaria de Vigilância em Saúde. Guia de vigilância epidemiológica do óbito materno. Brasília: Ministério da Saúde. 2009 [Acesso em 19 set 2015]. Disponível em:

http://svs.aids.gov.br/download/manuais/manual obito materno 2009.pdf

10 - Brasil. Ministério da Saúde. Secretaria de Vigilância em Saúde. Manual de vigilância do óbito infantil e fetal e do Comitê de Prevenção do Óbito Infantil e Fetal 2. 2009 [Acesso em 19 set 2015]. Disponível em:

http://bvsms.saude.gov.br/bvs/publicacoes/manual obito infantil fetal 2ed.pdf

11 - Brasil. Rede Interagencial de Informações para a Saúde - RIPSA. Indicadores básicos de saúde no Brasil: conceitos e aplicações. Brasília: OPAS; 2002.

12 - Brasil. Agência Nacional De Vigilância Sanitária. Serviços de atenção materna e neonatal: segurança e qualidade. Brasília: ANVISA; 2014.

13 - Brasil. Ministério da Saúde. Portaria n 1.020, de 29 de maio de 2013. Institui as diretrizes para a organização da Atenção à Saúde na Gestação de Alto Risco e define os critérios para a implantação e habilitação dos serviços de referência à Atenção à Saúde na Gestação de Alto Risco, incluída a Casa de Gestante, Bebê e Puérpera (CGBP), em conformidade com a Rede Cegonha. [Acesso em 19 set 2015]. Disponível em http://bvsms.saude.gov.br/bvs/saudelegis/gm/2013/prt1020 2905 2013.html.

14 - Brasil. Ministério da Saúde. Portaria Interministerial no 285, de 24 de março de 2015. Redefine o Programa de Certificação de Hospitais de Ensino (HE). [Acesso em 03 dez 2015]. Disponível em http://bvsms.saude.gov.br/bvs/saudelegis/gm/2015/prt0285 2403 2015.html.

15 - Brasil. Agência Nacional De Vigilância Sanitária. RDC n 63 de 25 de novembro de 2011. Dispõe sobre os Requisitos de Boas Práticas de Funcionamento para os Serviços de Saúde. Diário Oficial da União n²27, 28 nov. 2011, seção 01, p. 44 - 46.

16 - Fragata J, Sousa P, Santos RS. Organizações de saúde seguras e fiáveis/confiáveis. In: Sousa P. organizador. Segurança do Paciente: criando organizações de saúde seguras. Rio de Janeiro: Editora Fiocruz; 2014.

17 - Vincent C. Segurança do paciente: orientações para evitar eventos adversos. São Caetano do Sul - SP: Yendis; 2009. 